\title{
AC 2009-814: EDUCATIONAL OUTREACH MATERIALS FOR ERC STRUCTURED ORGANIC PARTICULATE SYSTEMS: A PROJECT OVERVIEW
}

Stephanie Farrell, Rowan University

Zenaida Otero Gephardt, Rowan University

Robert Krchnavek, Rowan University

Mariano J. Savelski, Rowan University

C. Stewart Slater, Rowan University

Eileen Batten, Rowan University

John Carroll, Rowan University

Christopher Del Vecchio, Rowan University

Adrian Kosteleski, Rowan University

Katie Ross, Rowan University

Tatsiana Sokal, Rowan University

Kathryn Whitaker, Rowan University

Sarah Wilson, Rowan University 


\title{
Educational Outreach Materials for ERC Structured Organic Particulate Systems: Project Overview
}

\begin{abstract}
Rowan University chemical engineering students and faculty are working in collaboration with the National Science Foundation (NSF) funded Engineering Research Center for Structured Organic Particulate Systems (C-SOPS) to create educational materials based on research being conducted on advanced pharmaceutical processing. The Center is hosted by Rutgers University and also involves Purdue University; the New Jersey Institute of Technology; and the University of Puerto Rico, Mayaguiez. The goal of the Center is to become a national focal point for developing structured organic particulate systems used in pharmaceuticals and their manufacturing processes. Rowan University has partnered as an outreach/education member institution. The goal of our interaction is to contribute to SMET education at both K-12 and college levels through the integration of C-SOPS research into detailed homework problem sets, illustrative examples, demonstrations and case studies. The curriculum modules will be tailored to specific age levels by varying the technical content level. Completed educational materials will be incorporated into the C-SOPS website for use by the members of the Center and faculty at other schools. The overall goal will be to expand the impact of the Center through the field of education and outreach.
\end{abstract}

\section{Introduction}

The NSF-sponsored Center for Structured Organic Particulate Systems (C-SOPS) is striving to become a focal point in pharmaceutical processing. Carefully planned out thrust areas and development programs tie together the overall goals of the Engineering Research Center (http://ercforsops.rutgers.edu/). The thrust areas describe the three major areas of research, which include: manufacturing science; composites structuring and characterization; and particle formation and functionalization. The areas of research can then be applied to the three development programs, which describe each of three test beds being created at the Center. Development Program I concentrates on the continuous manufacturing of pharmaceutical tablets. Utilizing a continuous process rather than a batch process could lead to increased tablet uniformity and stability, reduced production and labor costs and simplified scale up from experimental testing to full scale manufacturing [1]. Development Programs II and III focus on novel methods for drug delivery. Development Program II hopes to stabilize API nanoparticles in edible substrates [1]. The use of nanoparticles increases the surface area of the particles, resulting in higher bioavailability. Finally, Development Program III will use a system called drop-on-demand to layer API's on an edible substrate [1]. The system could be portable and compact for use in third world countries and military applications.

Rowan University partnered with the ERC-SOPS Center in 2008 to provide outreach and training components to support the educational mission of the Center. During our first year on the project, Rowan has worked with various constituency groups to implement certain projects that directly impact the Center's goals. 
This paper describes our initial efforts in this area. Our long term goals are:

- Train students who will be effective engineers and leaders in the manufacturing and research operations of the pharmaceutical and allied industries of the center.

- Train students for roles in education and in the agencies involved in regulating food and drug manufacturing operations.

- Bring the new Center research discoveries in engineered organic composite systems to enrich the existing engineering curriculum at both the undergraduate and graduate levels - Develop educational programs for industrial practitioners and foster alliances with industry in the education and outreach activities of the center.

- Design and promote experiential programs and pedagogical material for K-12 outreach recognizing diverse student and teacher backgrounds.

- Develop a suite of modular educational units for use by the various center constituents in formats that allows for efficient web-based dissemination.

These goals are consistent with the overall center vision and are an integral part of its mission to bring together cutting-edge research, technology transfer and next-generation training of the technical workforce.

To better reach the proposed goals, we assembled a group to highly qualified educators from the College of Engineering. Each faculty member has been working with undergraduate and graduate students to develop the educational materials.

The following sections summarize the various activities performed during the first year of the Rowan / ERC-SOPS Center partnership. These sections of the paper are organized by each area of educational methods developed for the ERC. Detailed examples of the outreach/educational materials will be presented in the final poster presentation.

\section{Curriculum Module Development in Structured Organic Particulate System Manufacturing}

This component of the ERC outreach effort is the development of problem sets for use as in-class examples and homework problems for chemical engineering and K-12 courses. Drs. C. Stewart Slater and Mariano Savelski led this aspect of the project with undergraduate students Christopher DelVecchio, Adrian Kosteleski, and Sarah Wilson. We worked with Rutgers graduate student liaisons, William Engisch and Frank Romanski, who provided feedback to the students. The goal of this work is to translate organic particulate systems manufacturing concepts and research being done the Center into educational materials that can be used at various levels. The problem sets, a.k.a. "curriculum modules," are tailored to specific age levels by varying the technical content level of the problems. Each curriculum module consists of a multi-part problem statement with a link to an ERC area, relevant literature references, and fully executed solution. Examples of problem content include basic scientific and engineering concepts such as, unit conversions, mass and energy balances, fluid/particle flow. The modules support a specific learning objective in a fundamental science or engineering area (e.g., material balances), while introducing students to a new concept (powder flow in pharmaceutical manufacture) and have a link to the ERC (specific thrust area/test bed). For example, the 
problem "Continuous Powder Flow in High Shear Mixers," is a problem on mass balance calculations on one process unit using two-component system. The problem introduces students to the concept of powder flows and powder blending equipment, and is linked to Test Bed 1. This problem is made more meaningful since a reference to an ERC publication, Portillo, Ierapetritou and Muzzio, Powder Tech., (2007) 1-10, allows students to explore the subject in greater depth. An example of a K-12 problem is "Fractions, Decimals, and Percentages in Medicine Tablets" is designed for basic mathematics audience to teach the concept of fractions and percentages, by using the components (API, binder, filler) in a common drug tablet. We have created over 20 preliminary problems sets. We have additional problems under development. The draft problem sets are indexed by grade level of the problem as well as problem linkage to ERC area. When completed this SOPS curriculum guide will make it easy for an instructor to pick problems to integrate into their course.

The plans for 2009 are to pilot test these problems in some of our courses at Rowan and obtain feedback/assessment for improvement of the problem statements and solutions. We also plan to develop new problems in other ERC areas as well as for other courses in the chemical engineering curriculum. We intend to provide more animation and artwork for the K-12 problem sets. The students will benefit from close interaction with graduate student liaisons from the ERC so that we can be provided with background material on research activities of the Center as well as feedback on the technical content of the educational materials we develop. An example of a completed problem set will be presented in the poster presentation.

\section{Synthesis of Nanoscale Particles}

Dr. Robert Krchnavek has led the efforts in this area along with undergraduate student John Carroll. A series of laboratory experiments suitable for high school students, are being developed. These laboratory experiments reflect the importance of nanoscale drug particles. Our experiments intend to demonstrate (i) the enhanced solubility of a nominally insoluble particle by creating a nanoscale version of it, (ii) demonstrate the various methods of achieving nanoscale particles that are relevant to the bionanotechnology industry. Of course, the greatest challenge in this endeavor is to create laboratory experiments with dramatic impact for the students under the constraints that high schools have very limited resources. An example of one of these experiments will be presented in the poster.

\section{Controlled Release Systems for Oral Drug Delivery}

During the first year, Dr. Stephanie Farrell has been working with undergraduate students Eileen Batten and Kathryn Whittaker in the development of an educational module to introduce students to the design, preparation, characterization, and analysis of drug delivery systems. The module, Matrix Tablet Formulation and Characterization, is based on the design and manufacture of an oral formulation for controlled caffeine delivery. During the preparation of this tablet, the active ingredient is mixed with a hydrophobic matrix, and compressed into tablet form using a standard method such as the direct compression. Drug, matrix, and process parameters affect the tablet's physical properties which include hardness, disintegration, and dissolution. Drug release kinetics from tablet matrices commonly follow a square-root-of-time dependence first described by Higuchi [2]. 
The educational module will teach students about the design and production of drug matrices in tablet form for caffeine delivery as a specific example. The module is based on an experiment previously developed at Rowan [3]. The materials developed so far include:

- A Powerpoint presentation on introduction to drug delivery This presentation introduces methods of drug delivery using polymeric systems. The advantages and disadvantages of controlled drug delivery are described.

- A introduction to drug release from matrices Development of the Higuchi Model is presented for a lower level college audience.

- A laboratory handout for an experiment on tablet production In this experiment, students produce tablets using caffeine as the active pharmaceutical ingredient. The tablets are formulated using a hydrophobic microsphere resin for controlled release, and the release follows Higuchi kinetics.

- Photographs of tablet production equipment

Diagrams and photographs have been produced to show the tablet press and its components.

- A problem set with solutions

Several problems have been developed for high school and lower-level college students. The problems focus on concepts related to tablet formulation, fraction of drug released, rate of drug released, and therapeutic concentration. Many of the problems relate to the caffeine tablet experiment and require analysis of experimental data.

Several of these materials will be distributed at the conference in Austin.

\section{Particle Properties and Powder Mixing Experiments throughout the Curriculum}

This component of the ERC outreach effort is being developed by Dr. Zenaida O. Gephardt with undergraduate students Michael Raymond, Lindsay Poole, Christopher DelVecchio, Tatsiana Sokal, and Katie Ross.

A V-mixing laboratory experience has been designed. Students will study the effect of mixing time and loading configuration in a statistical design of experiments program. Students will be exposed to experimental design strategies. The experiments and data analysis will be conducted over multiple class periods. A $5 \mathrm{~L}$ constant frequency V-mixer was purchased. It will be used for laboratory experiences in courses, Engineering Clinic projects and research. Figures 1a and b show the mixer and the loading operation for a mixing experiment. 


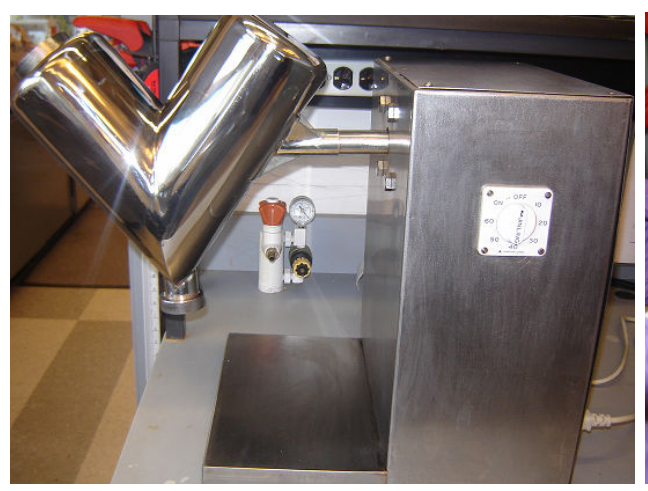

Figures $1 \quad$ (a) 5 L V-mixer

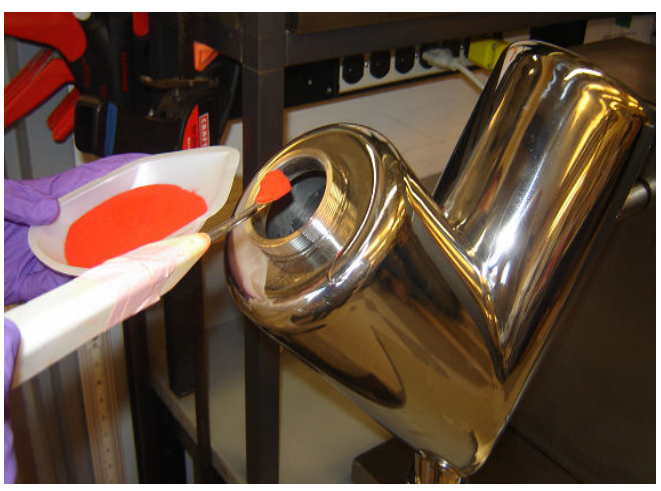

(b) Loading mixer for experiment

Factorial and response surface Box-Behnken experimental designs were developed. Variables studied included particle size and particle size difference, mixing time and loading configuration. The difference in loading for each mixer arm was also investigated. Figure 2 is a qualitative schematic illustrating a Box-Behnken Design for three variables; mixing time, particle size difference and loading configuration. The circles indicate experimental conditions and the vertices are the minimum and maximum values of the variables investigated. In the case of loading configuration, the experimental conditions refer to specific configurations (i.e. top/down, side by side). Preliminary results are in agreement with most of the literature and indicate that particle size is a key variable affecting mixing quality for most applications. However, initial results also indicate that some of the interactions not previously considered are also significant in the determination of mixing quality.

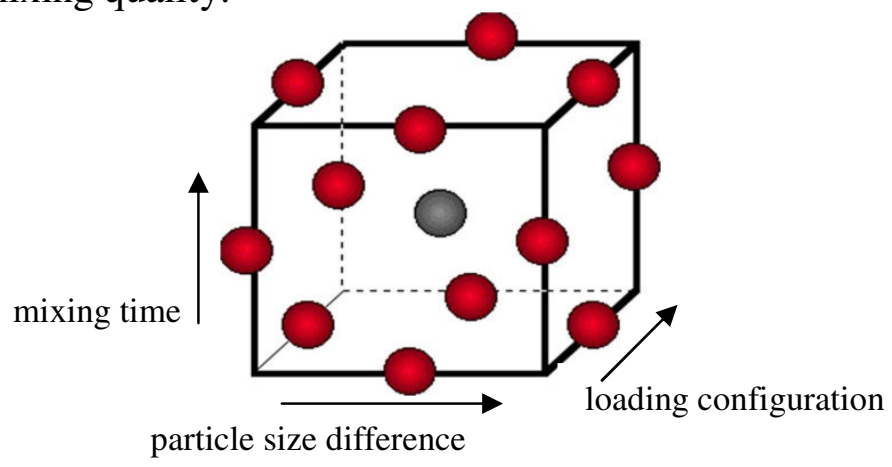

Figure 2: Qualitative schematic of Box-Behnken Design

A spectrophotometric technique was devised to measure mixing quality for these experiments. Different color silica particles were used so that students could easily distinguish among different mixtures. After the particles were mixed at specific conditions, a sample $(2 \mathrm{~g})$ of the particle mixture was placed in $25 \mathrm{ml}$ of hexane. The dye dissolved in the hexane, and a spectrophotometer was used to detect the absorbance of the liquid at the wavelength corresponding to the dye color. Red particles with compounded dye and yellow particles were used (Figure 1b). The red dye was not soluble in hexane, thus only yellow was detected using the spectrophotometer. Tracking only one color simplified the spetrophotometric analysis since color mixtures did not have to be considered. Statistical analysis of the data for different samples in different locations in the mixer, and the Poole index were used to assess mixing quality. 
These experiments afford students the opportunity to study particle and powder mixing with applications. In addition, students are exposed to experimental design and to the statistical measures of mixing quality. Standard deviation measures and the Poole index were used to measure mixing quality. This work is ongoing. A Spring 2009 project has been developed to complete the first phase of this investigation. Additional investigations involving different types of particles and combining mixing studies with other operations such as tablet formation are being considered.

A series of highly visual demonstrations illustrating particle properties has been designed, constructed and used in a course. These demonstrations will be used in outreach activities for high school and middle school teachers during the Spring 2009 semester. In addition, they will be used for summer programs aimed at high school and middle school students. All demonstrations can be constructed from a variety of materials and in different sizes. They can be adapted for use in K-12 activities as well as upper level technical courses. Details on these demonstrations and experiments will be provided in the poster presentation.

\section{Development of a New Course in Particle Technology}

Dr. Zenaida Gephardt has developed a new graduate/senior level course Fundamentals of Particle Technology (senior) and Advances in Particle Technology (graduate). This course is unique in that it embeds different levels of technical material that are appropriate for seniors and graduate students. Graduate students have more comprehensive and complex projects and additional homework.

The course includes

- standard seminar style presentations

- hands-on experiences using the demonstrations described below

- a fluidization experiment in which students obtained the minimum fluidization velocities in laboratory scale fluidized beds and estimated the average particle size using their data.

In addition, students

- completed a critical literature review of specific powder and particle technologies for pharmaceutical applications. Graduate students presented their results to the class.

- completed a final project including a written final report and a presentation to the class. Students were assigned a refereed journal article to review and critique using concepts presented in class. Their written report included calculations and substantiation for their review.

The course included a balance of individual and group assignments, homework and in-class problem solving. Introduction to Particle Technology by Martin Rhodes was the textbook used. In addition, supplementary materials and relevant journal articles were integrated throughout the course. The course included

- $\quad$ basic particle properties and size analysis

- mixing and segregation

- size reduction (breakage) and enlargement

- transport of slurries, colloids and fine particles 
- flow through packed beds and fluidization

- particle and powder technology applications with a focus on pharmaceutical applications

- health effects and fire and explosion hazards in fine powder operations

Students were guided through the development of their own experimental strategies and manuals as well as relevant data analysis. Pharmaceutical applications such as V-mixers, tablet formation and capping were presented and discussed.

\section{Summary}

By incorporating research being conducted at the C-SOPS into curriculum modules, students will be educated on novel techniques being used in pharmaceutical processing. The work focuses on students at both a K-12 and collegiate level. Developed modules will be integrated into the CSOPS website for distribution to faculty within the Center and other schools. Overall, the goal of the educational outreach program is to expand the impact of the Center through increasing student interest in pharmaceutical engineering.

\section{Acknowledgments}

This project is supported by National Science Foundation - Engineering Research Center grant \# 0540855. We acknowledge the guidance of ERC faculty, Dr. Fernando Muzzio, Rutgers University, ERC Technical Director and Dr. Henrick Pedersen, Rutgers University, ERC Education Director and the input of graduate students Frank Romanski and Bill Engisch of Rutgers University in reviewing the course materials.

\section{References}

1. http://www.erc-assoc.org/annmtg/2006_meeting_files/HO\%20PSIII\%20Muzzio.ppt

2. Higuchi, T., "Rate of release of medicaments from ointment bases containing drugs in suspension", J. Pharm. Sci., 50 (10), p. 874-5, 1961.

3. Farrell, S., R. Hesketh, M.J. Savelski, and C.S. Slater, Drug Delivery Experiments in the ChE Curriculum, Proceedings of the 2005 ASEE Conference, Session 1526, June 2005. 